\title{
Directoras y directores escolares frente a la Ley de Inclusión Escolar en Chile: entre compromiso, conformismo y resistencia
}

\author{
School Principals Faced to the School Inclusion Law \\ in Chile: Between Engagement, Conformity and \\ Resistance
}

\author{
María Teresa Rojas Fabris ${ }^{1}$, Natalia Salas ${ }^{2}$ y José Ignacio Rodríguez ${ }^{3}$ \\ ${ }^{1}$ Universidad Alberto Hurtado, Chile. \\ ${ }^{2}$ Universidad Finis Terrae, Chile. \\ ${ }^{3}$ Universidad Mayor, Chile.
}

\section{Resumen}

El artículo comunica los resultados de una investigación realizada en Chile sobre las disposiciones de directoras y directores frente a la Ley de Inclusión Escolar, regulación que promueve la desegregación escolar y que comenzó en 2016. Considerando el enfoque del liderazgo inclusivo y de justicia social, el estudio se construyó a partir de una encuesta en línea a 157 directoras/es de cuatro ciudades. Dado su carácter exploratorio, se siguió un diseńo cuantitativo no experimental con un alcance descriptivo. Los resultados muestran que las y los directores comparten los principios de esta política, pero prevén que tendrán problemas por la falta de capacitación de las y los profesores y por las tensiones que supone implementar políticas de inclusión en un sistema escolar que mide la calidad a partir de pruebas estandarizadas. Se propone que las y los directores manifiestan compromiso con los principios de la política, pero a la vez un conformismo y resistencia con sus implicancias más específicas, pues no aprecian que esta modifique las lógicas de funcionamiento de sus escuelas.

Palabras clave: Inclusión escolar; liderazgo educativo; justicia social; desegregación, política educativa.

\section{Correspondencia a:}

MaríaTeresa Rojas Fabris.

Facultad de Educación, Universidad Alberto Hurtado.

Almirante Barroso 10, Santiago, Chile.

mtrojas@uahurtado.cl

Agencia de patrocinio: Centro de Desarrollo y liderazgo Escolar CEDLE.

C 2021 PEL, http://www.pensamientoeducativo.org - http://www.pel.cl

ISSN:0719-0409ＤDI:203.262, Santiago, Chile doi: 10.7764/PEL.58.1.2021.6 


\section{Abstract}

The paper presents the results of a study carried out in Chile on the attitudes of principals to the School Inclusion Law, legislation that promotes the desegregation of schools and which went into force in 2016. Considering the approach of inclusive leadership and social justice, the study was conducted based on an online survey of 157 principals from four cities. Because of its exploratory nature, the study followed a non-experimental quantitative design with a descriptive scope. The results show that principals agree with the provisions of this policy, but they anticipate that they will have problems due to the lack of training of teachers and the tensions involved in implementing inclusion policies in the context of a school system that measures quality based on results on standardized tests. Therefore, school principals show commitment to the principles of the policy, but, at the same time, they demonstrate conformity with and resistance to its more specific implications, since they do not believe that it structurally changes the operating logic of their schools.

Keywords: School inclusion; educational leadership; social justice; desegregation, educational policy

La Ley de Inclusión -LIE - es una política educativa que comenzó su implementación el año 2016 en Chile. Su foco apunta a eliminar las barreras de mercado que han segregado a la educación chilena estas últimas décadas. Específicamente, busca regular el mercado educativo chileno que se gestó a inicios de los años 80 durante la dictadura de Augusto Pinochet y que se ha prolongado hasta hoy. Este mercado se organizó con base en una oferta gratuita, asentada en la gestión de las municipalidades, y una privada, con subvención del Estado, que podía lucrar y, además, obtener un copago de las familias. Diversos estudios muestran que Chile es un país con altos índices de segregación escolar (OECD, 2018; Murillo y Martínez-Garrido, 2017; Santos \& Elacqua, 2017; Arcidiácono et al., 2014; Bellei, 2013; Elacqua, 2012; Mineduc, 2012). Las medidas que propone la LIE incorporan las recomendaciones de la OCDE para hacer frente a la segregación escolar, mantenido un balance entre la libertad individual de la elección de escuela, y la igualdad social necesaria para asegurar sociedades menos desiguales y más cohesionadas (OECD, 2012). Estas medidas son: a) eliminar la posibilidad de cobro por matrícula y mensualidad a las familias, b) prohibir la selección de estudiantes en todos los niveles desde prekínder hasta $4^{\circ}$ medio, c) impedir el retiro de utilidades por parte de los sostenedores de las escuelas, y d) restringir la expulsión de estudiantes solo a aquellos casos de violencia extrema que afecten gravemente la seguridad de la comunidad escolar (BCN, 2014). Esta política afecta a todos los establecimientos educacionales, públicos o privados, que reciben aportes del Estado, los que representan 91,7\% del total de escuelas de Chile (Mineduc, 2017), quedando fuera de la norma aquellas escuelas privadas que no son subvencionadas (con excepción del punto d). Además, establece ciertas excepciones respecto de la selección escolar, flexibilizando el criterio cuando se trata de establecimientos con un proyecto educativo particular (deportivo, artístico, etc.) o de establecimientos denominados liceos emblemáticos, que cuentan con una cuota de $30 \%$ para elegir a sus estudiantes desde el séptimo grado escolar.

La agenda de la LIE deviene de las demandas del movimiento estudiantil de 2011 en contra de las exclusiones y consecuencias estratificadoras del mercado escolar chileno, como también de la degradación de la escuela pública en el actual modelo de gobernanza. El amplio apoyo ciudadano que caracterizó en este período al movimiento estudiantil impulsó la reforma educacional del segundo gobierno de Michelle Bachellet (2014-2018), que hizo eco de las demandas por la gratuidad, el fin al lucro, y la segregación (Castillo, 2017). 
En consideración a los temas que regula la LIE, es posible considerarla una política de desegregación que, por una parte, promueve la mixtura social en los establecimientos, pues elimina gradualmente las barreras socioeconómicas para acceder a todas las escuelas (Ayed, 2015); por otra, favorece la justicia escolar, ya que distribuye equitativamente las posibilidades de acceder a un establecimiento subvencionado por el Estado con la finalidad de construir comunidades diversas en las que no existan brechas de discriminación (Ryan, 2006).

Desde el punto de vista de directoras y directores de escuela, estos cambios son un desafío para su gestión y liderazgo, pues la LIE no solo modifica prácticas específicas de las escuelas al centralizar los procesos de admisión, desprivatizar el origen de los recursos económicos e impedir el retiro de utilidades, sino que además tensiona la cultura escolar al modernizar la noción de inclusión escolar en el sistema educativo, potenciando la aceptación de la diversidad estudiantil, y alejándose del modelo de integración ligado exclusivamente a la atención de las necesidades educativas especiales. Desde esta perspectiva, resulta clave conocer qué opinan las y los directivos escolares sobre una política de esta naturaleza, para comprender sus disposiciones frente a una regulación que fomenta la desegregación y, al mismo tiempo, analizar las dificultades que viven o avizoran en el marco de las nuevas exigencias de esta política pública. Este estudio parte de la base de que los modos en que los directores y directoras de escuelas enfrentan una política que promueve la desegregación y la justicia social inciden en la traducción que realicen de esta y en la manera en que la comunicarán en la escuela. Conocer, por tanto, cuáles son las disposiciones de directoras y directores en los inicios de la implementación de esta política permite evidenciar la relación entre las políticas públicas y las formas de apropiación que realizan de ellas los actores escolares.

\section{Marco teórico}

\section{Liderazgo inclusivo para promover la desegregación y la justicia social}

Durante las últimas décadas, las escuelas han visto emerger una creciente diversidad al interior de sus aulas. La población de estudiantes se ha transformado en un grupo crecientemente heterogéneo en términos de nacionalidades, etnias, capacidades, idioma, nivel socioeconómico y género. La existencia de esta diversidad ha interpelado a los sistemas educativos tradicionales para responder a las demandas por justicia y reconocimiento de los sujetos. En este escenario, el liderazgo para la inclusión social sugiere que los directivos de las escuelas se planteen críticamente frente a la desigualdad social producida por el sistema escolar (Oplatka \& Arar, 2015), lo que significa ceder en la centralidad de los logros académicos exigidos por los sistemas escolares y otorgar más tiempo y relevancia al desarrollo moral y ético de los sujetos. En la misma línea, Ryan (2006) señala que el liderazgo se ha alzado como un elemento relevante a la hora de realizar esfuerzos para mejorar las escuelas, y que debiera enfocarse más en propósitos morales, como la justicia social. Los desafíos de la escuela actual están relacionados con la ciudadanía y moral de niñas y niños, para favorecer mejores condiciones de vida para poblaciones crecientemente multiculturales. El liderazgo escolar orientado a la inclusión y la justicia social coloca en el centro de sus objetivos formativos el trabajo de formación ciudadana y democrática al interior de una escuela (Ryan, 2006).

Oplatka y Arar (2015) presentan una propuesta para el liderazgo directivo orientado a la inclusión y la justicia social, cuya base está en la constitución de un equipo directivo con conciencia crítica sobre la opresión, exclusión y marginalización; en otras palabras, un equipo que posee una opinión y un análisis sobre cuáles son las prácticas institucionales que favorecen a ciertos grupos sobre otros en una escuela o en un territorio. Esta conciencia, según los autores, permite promover una cultura escolar que se organiza en torno a fines tales como el valor de la justicia en relación con las interacciones personales y con la persona en sí misma en la escuela, para permitir que la voz de todos y todas sea escuchada; el respeto a una amplia variedad de expectativas culturales y sociales, el aprecio a la participación de las comunidades de los estudiantes y, de manera gradual, ir involucrando a profesores y estudiantes, entre otros. 
Por otro lado, Capper y Young (2014) identifican aquellas áreas de acción de los directivos de una escuela que promueven la creación de comunidades más inclusivas. En primer lugar, apuntan a consolidar un acuerdo sobre el significado de la inclusión/integración, que debiese transformarse en el elemento guía de todas las políticas y prácticas destinadas a eliminar las desigualdades educativas al interior de la escuela. Este consenso en el uso y sentido del término inclusión es especialmente relevante para tener horizontes formativos claros y compartidos por toda la comunidad. Lo segundo es transformar el aprendizaje de las y los estudiantes en el objetivo principal del trabajo directivo. Las autoras señalan que, si bien los logros académicos son solo un aspecto del bienestar estudiantil y que la práctica educativa debiese estar relacionada con la transformación de la comunidad, a la larga es necesario formar estudiantes que sean capaces de leer, escribir, comunicarse al nivel esperado para su edad, o incluso más, para que sus oportunidades educacionales y de vida sean considerables. La formación ciudadana es indisociable de una formación académica que permita que los sujetos movilicen competencias similares en contextos diversos. La tercera área de acción es conocer el amplio rango de diferencias de sus estudiantes y las intersecciones de estas diferencias, como las capacidades diferentes, raza, orientación sexual, género, diversidad lingüística y, por último, también señalan como importante comprender la promoción de la justicia como un esfuerzo colectivo entre los directivos de la escuela, equipos de profesores, profesoras y miembros de la comunidad. El liderazgo inclusivo se orienta, por tanto, explícitamente a la consecución de la justicia social.

Las premisas anteriores son fundamentalmente normativas, pues proponen ciertos propósitos y metas del liderazgo orientado a la justicia social. No obstante, en términos empíricos, diversas investigaciones dan cuenta de las tensiones que enfrentan los directivos en el empeño de contribuir a la inclusión y la justicia social en sus escuelas. Archambault y Richer (2014), por ejemplo, detallan la implementación de un programa de apoyo (Supporting Montreal Schools Program, SMSP) a las escuelas de la provincia de Quebec, Canadá, cuyo objetivo era terminar con las injusticias - dirigiendo la enseńanza y el aprendizaje contra los prejuicios y creencias racistas y clasistas- y mejorar los resultados de aprendizaje. Los autores identifican que la mayoría de las directoras y directores no poseen una conceptualización específica sobre la inclusión y la justicia social. Estos reconocen que en su formación y en su carrera directiva no han tenido posibilidades ni condiciones para reflexionar al respecto. Otro estudio identifica que los directivos que poseen una conciencia política orientada a promover mayor inclusión y justicia dedican mucho tiempo a mantener conversaciones difíciles sobre el sentido de las acciones pedagógicas, crear conciencia entre los docentes, identificar barreras para implementar ideas alternativas, comprender y apreciar las diferencias, entre otras (Bogotch \& Reyes-Guerra, 2014). En estos casos, resulta más visible capturar un discurso orientado a la inclusión y la justicia en la escuela, más que un plan de gestión escolar que proponga una lógica diferente de la organización escolar.

En esta misma línea, Theoharis (2010) destaca las importantes barreras o resistencias a las que se enfrentan los directivos de las escuelas, relativas a las reacciones dentro de la escuela y desde el distrito y la administración. No siempre existe concordancia entre los objetivos del director o directora de la escuela y las prioridades de quienes administran el sistema escolar de un determinado territorio. Algo similar observa Ryan (2010), al constatar que la instauración de prácticas inclusivas no está exenta de dificultades e impedimentos, como hacer frente a sistemas altamente jerárquicos, la resistencia de colegas, padres y estudiantes; ambientes de cuasi-mercado y altas cargas de trabajo.

El estudio de Ryan (2010) ayuda a reconocer que la promoción de la inclusión y la justicia social es un proceso complejo que requiere condiciones internas en la escuela, pero también el alineamiento de los organismos públicos que administran las escuelas y de las políticas que las regulan. En este sentido, el autor sostiene que resulta altamente incompatible promover políticas de inclusión en sistemas educativos que miden la calidad mediante pruebas estandarizadas y establecen premios y castigos por ello. Este es un ejemplo real de las diferencias que pueden movilizarse entre los líderes de los distintos niveles de gestión del sistema escolar, una tensión que atraviesa buena parte de los sistemas escolares en la actualidad. 
Existe en la literatura una aproximación normativa al liderazgo inclusivo orientado a la justicia social que prescribe y recomienda acciones y disposiciones de directoras, directores y líderes escolares en torno al tema. Por su parte, los estudios de caso, situados en distintos contextos, permiten identificar las distancias entre la prescripción y la puesta en práctica del liderazgo inclusivo. En este punto, se hace necesario mirar específicamente el peso de las disposiciones y opiniones de los directivos en los procesos de puesta en práctica de acciones que pueden promover mayor inclusión y justicia en una escuela.

\section{Disposiciones de directoras y directores frente a una política de desegregación que}

\section{promueve la justicia social en la escuela}

La implementación de las políticas educativas está condicionada por una serie de factores, entre ellos los recursos materiales y profesionales para su realización, los sistemas locales de apoyo a las escuelas, su vinculación con otras políticas que afectan a la escuela, la capacidad del gobierno central y los organismos intermedios de comunicar sus propósitos y, además, por la lectura y traducción que los propios actores escolares realizan de la política (Draelants, 2018). En este último sentido, directoras y directores son actores claves en la comprensión de las políticas educativas. Los y las directores/as traducen las políticas en prácticas y acciones dependiendo las posiciones que poseen y los recursos que manejan. Molla y Gale (2018) proponen que los directivos enfrentados a implementar políticas de promoción de la equidad y la justicia escolar movilizan distintas estrategias y tácticas para traducirlas al lenguaje de la gestión escolar y, a la vez, para trabajarlas con sus comunidades. Los autores, con base en estudios empíricos realizados en escuelas australianas, sostienen que directoras y directores traducen la política con disposiciones diferentes, que se sostienen en tácticas específicas y que dependen de sus contextos de práctica. Al respecto, proponen tres disposiciones características: conformismo, compromiso o resistencia.

Existe conformismo frente una política cuando esta se asume con pasividad o resignación, es decir, cuando directoras y directores movilizan una lógica de conformismo (Ball, Maguire, \& Braun, 2012). Esta disposición suele asociarse a directivos que trabajan en contextos muy desfavorecidos y que consideran que cuentan con recursos insuficientes para implementar las leyes basadas en agendas de justicia social, más aún si no están asociadas a nuevos presupuestos. Puede existir un relativo acuerdo con los principios de una política, pero es más importante la percepción de que no tendrá mayor impacto en la calidad de aprendizajes y de vida de los y las estudiantes. En estos casos, las directoras y directores asumen las nuevas regulaciones como un trabajo más en sus tareas, pero sin la convicción de que la política genere cambios importantes en su contexto. Capper y Young (2014) proyectan que en estos casos es probable que quien dirija la escuela no cuente con una reflexión sobre los alcances de la justicia social y, por lo tanto, no puede liderar procesos inclusivos porque no ha integrado una red de conceptos que le permitan trabajarlos en su gestión.

Por otra parte, una disposición asociada al compromiso refiere a un cierto alineamiento entre los principios de una política y los valores o juicios políticos de un director o directora. Es probable que, si el directivo cuenta con algún tipo de formación académica en temas de inclusión y justicia social, este alineamiento sea más favorable y se facilite. El compromiso no significa que la directora o director acepte todo lo que está escrito en la política, sino más bien que los principios que promueve se ajustan a sus creencias previas y a lo que le resulta factible y pertinente de implementar en su escuela (Lipsky, 2010). Existe cierta coincidencia entre la propuesta de la política pública y la forma en la que el director o directora concibe su trabajo como líder escolar, lo que favorece su identificación con la agenda de inclusión o justicia del Estado.

Finalmente, la disposición resistente o crítica alude a las sospechas que pueden provocar en las directoras y directores las contradicciones entre los principios que promueve una política de justicia social y los recursos que otorga para realizarlos. Una política que promueve una retórica desegregadora o inclusiva puede generar nuevas 
frustraciones si la escuela no tiene recursos ni redes para implementarla o movilizar los efectos esperados. En este caso, a diferencia de la disposición conformista, el director o directora lee la política como una normativa que le resulta imposible de implementar o cuya puesta en práctica puede dilatar para más adelante, porque no se ajusta a la realidad de su escuela. En este tipo de disposición se refuerza la postura de que las leyes son imposiciones de arriba hacia abajo, es decir mandatos de las autoridades del estado a las escuelas, que excluyen las percepciones de los actores escolares y son insensibles a sus verdaderas prioridades (Molla \& Gale, 2018).

\section{Metodología del estudio}

Dado su carácter exploratorio, el estudio siguió un diseño cuantitativo no experimental con un alcance descriptivo, justificado por el poco tiempo de implementación de la LIE. El trabajo de recolección de los datos se realizó en 2018, después de dos años de los inicios de la implementación de esta regulación.

La muestra se seleccionó mediante el método de muestreo estratificado simple. Para ello, se utilizó un criterio de inclusión por conveniencia, incorporando a todas las escuelas pertenecientes a las capitales de las regiones del país que -al momento de la realización del estudio-ya se habían incorporado al nuevo sistema de admisión (SAE) que contempla la Ley de Inclusión Escolar (LIE). Estas capitales son Iquique, Coquimbo, Puerto Montt y Punta Arenas. Posteriormente, considerando a cada ciudad como un estrato, se procedió a obtener una muestra representativa de las 273 escuelas que representaban el universo de estudio. Con un nivel de confianza de 95\%, un margen de error de 5\%, y suponiendo una varianza población de 50\%, la muestra requerida ascendió a 160 escuelas distribuidas en cada estrato según las proporciones del universo muestral. Finalmente, 157 directoras y directores de escuela respondieron completamente la encuesta. La encuesta se aplicó en línea.

Para evidenciar las disposiciones de directoras y directores respecto de la LIE, la encuesta consideró cinco dimensiones: 1) nivel de acuerdo con los principios de la ley, 2) acciones implementadas a partir de la aplicación de la ley, 3) percepción sobre el perfil de estudiante que ha ingresado mediante el nuevo sistema de admisión, 4) nivel de participación de la comunidad escolar, y 5) obstáculos y necesidades para implementar la LIE.

La encuesta fue validada por tres expertos que emitieron juicios para aclarar sus contenidos. Luego, se realizó una prueba piloto con cuatro respondientes, que obtuvo una alta confidencialidad (alfa de Cronbach sobre 0,8 ).

Finalmente, el análisis de resultados se basó en el desarrollo de estadística descriptiva, mediante la cual se exploraron las principales tendencias en la información con el objeto de determinar aquellos patrones relevantes para la investigación. Estos análisis se realizaron a través del paquete estadístico SPSS 22.0.

\section{Resultados}

\section{Caracterización de la muestra}

De los 157 directoras/es participantes en la encuesta, 21\% pertenece a la ciudad de Iquique, 22,3\% a Coquimbo, 37,6\% a Puerto Montt, y 19,1\%, a Punta Arenas. La mayor parte pertenece a establecimientos públicos municipales o administrados por servicios locales de educación (72\%), y 28\% a escuelas particulares subvencionadas por el Estado.

En términos de género, 66 (42\%) de los encuestados eran hombres y 91 (58\%), mujeres. La mayor parte ha ejercido funciones directivas por más de siete años (59,2\%), y solo 18,5\% ha ejercido funciones de este tipo por menos de tres años.

Respecto de su formación, 80,9\% de ellos ha finalizado estudios de posgrado en el área de administración, gestión o liderazgo directivo. Sin embargo, solo 19,1\% seńala tener algún tipo de formación en temáticas relacionadas con la inclusión escolar. 


\section{Alto acuerdo frente a los principios que promueve la Ley de Inclusión}

Consultados sobre el nivel de adherencia a los principios fundamentales que promueve la Ley de Inclusión, directoras y directores señalaron mayoritariamente estar de acuerdo con ellos. Un 91,1\% señaló estar de acuerdo con el término del lucro, $84,7 \%$ con el fin de la selección, y 78,3\% con el fin del copago, dando cuenta de una amplia adherencia a los postulados básicos de la nueva regulación. Por otro lado, respecto de la limitación a la expulsión arbitraria, 69,4\% está muy de acuerdo/de acuerdo con esta medida. Esto se relaciona con la percepción de que la normativa permitirá un sistema educacional más equitativo, diverso y respetuoso de las diferencias entre los estudiantes (77,1\% está muy de acuerdo/de acuerdo). Es decir, los postulados generales de la ley cuentan con la aceptación de los directivos consultados.

No obstante, como lo indican los datos, existe un grupo minoritario más reticente a ciertos componentes de la nueva regulación. Específicamente, hay un 36\% de directoras y directores de escuelas particular subvencionadas en desacuerdo con la eliminación del copago que hacen las familias, y 18\% de los directivos no está a favor de la prohibición de procesos de admisión selectivos.

\section{El nuevo sistema de admisión escolar favorece la inclusión y la equidad}

En torno al nuevo sistema de admisión escolar (SAE), directoras y directores toman posición respecto de sus efectos en la equidad educacional, la libertad de elección de los padres, la meritocracia, la calidad, y la libertad de emprender proyectos educacionales. Sobre lo anterior, un porcentaje importante de directoras y directores piensa que el nuevo sistema de admisión reduce la libertad de las familias de elegir el colegio que desean para sus hijos (56,1\%). Sin embargo, al mismo tiempo, 81,5\% opina que este nuevo sistema comprendido en la ley contribuye a implementar un proceso de admisión que no discrimina por el origen social ni por las capacidades de los estudiantes.

En la encuesta se consultó además por la opinión general que tenían las y los directoras/es respecto del SAE, siendo mayoritarias las opiniones positivas: $66 \%$ cree que este sistema garantiza la inclusión y el respeto a la diversidad de los sujetos, y $61 \%$ cree que respalda la no discriminación de las familias. Entre quienes tienen opiniones contrarias, aunque son minoritarias, sus preocupaciones se centran en un eventual efecto negativo sobre los estudiantes más talentosos y esforzados (10\%), el perjuicio en la calidad académica de la escuela (9\%), y sobre posibles trabas para desarrollar proyectos educacionales diversos (6\%).

\section{¿A quiénes incluye la Ley de Inclusión Escolar?}

Las directoras y directores creen que el nuevo sistema de admisión (SAE) que propone la LIE significará el aumento en su escuela de estudiantes con diversidad cognitiva y necesidades de aprendizaje ( $90 \%)$. Sin embargo, otras categorías de identidad son también observadas, tales como: estudiantes extranjeros hispanohablantes (51\%), estudiantes pertenecientes a un nivel socioeconómico más bajo (39\%), de diversas culturas religiosas (34\%), con diferentes orientaciones sexuales (26\%), con discapacidad física-motora visual o auditiva (25\%), o con lengua materna diversa (18\%). Tan solo $8 \%$ indica haber matriculado estudiantes de nivel socioeconómico más alto luego de la aplicación del SAE.

Algunos fenómenos son relevantes a la hora de considerar los posibles efectos de la LIE en la composición estudiantil de las escuelas. Por ejemplo, 49,5\% de los directivos piensa que la LIE fomenta que las y los estudiantes de las escuelas públicas (administradas por municipalidades o servicios locales de educación) migren hacia las escuelas particulares subvencionadas. También es interesante considerar la opinión que los directivos tienen de 
los apoderados de sus escuelas. Un 55,4\% de las directoras y directores considera que las familias sí valorarán elegir escuelas que posean mixtura social, versus $22,9 \%$ que cree que las familias rechazarán la mixtura que promueve la LIE y tratarán de cambiar a sus hijos a escuelas que tengan menos estudiantes vulnerables.

\section{La Ley de Inclusión y su impacto en los resultados académicos}

Es interesante observar que la mayoría de los directivos no consideran que la Ley de Inclusión tendrá mayor impacto en la calidad de los resultados académicos de su escuela, medidos mediante la prueba nacional estandarizada (SIMCE). Solo $14 \%$ cree que sus resultados disminuirían, y un grupo no menor $(22,3 \%)$ piensa que es probable que sus resultados mejoren.

\section{Las primeras decisiones en torno a la Ley de Inclusión Escolar}

Consultados las directoras y directores sobre las primeras medidas adoptadas en el marco de la implementación de la Ley de Inclusión, los encuestados declaran que han informado a la comunidad escolar sobre el inicio de la gratuidad $(83,8 \%)$ y no realizaron cobro alguno por concepto de mensualidad a las familias $(70,3 \%)$. Por otro lado, respecto del uso de los recursos económicos que recibirá la escuela, la mayoría declara que los invertirá en la contratación de profesionales que apoyen el área psicopedagógica (72\%) y psicosocial (48\%), además de materiales educativos (64\%). Estas opiniones podrían dar cuenta nuevamente de que los directivos asocian esta ley de desegregación con el probable aumento de niños y niñas con necesidades educativas especiales.

\section{Condiciones del trabajo docente: obstáculo para liderar la inclusión en la escuela}

En relación con la percepción de obstáculos para una adecuada implementación de la Ley, los directivos consultados consideran que sus profesores no están capacitados para gestionar la diversidad social del alumnado (64\%), que carecen de tiempo destinado a planificar acciones inclusivas colaborativamente (66\%), y plantean que no existe coherencia entre el sistema de evaluación nacional (SIMCE) y las propuestas de la Ley de Inclusión (57\%).

Desde esta perspectiva, la formación en diversidad aparece como una de las principales necesidades para su implementación. Un 66\% declara necesitar formación en inclusión, y 43\% solicita formación en liderazgo inclusivo para el equipo directivo. A su vez, un alto porcentaje de directivos (66\%) manifiesta requerir menor presión en torno a los resultados en pruebas comparativas, tanto a nivel nacional como internacional.

Finalmente, cabe destacar que las directoras y directores encuestados declaran que su principal demanda al gobierno es contar con mayor orientación pedagógica respecto de la organización de la enseńanza en contextos de diversidad. Se menciona la necesidad de disminuir la cantidad de niños por aula (34\%), la adquisición de recursos didácticos y materiales pedagógicos adecuados (46\%), inversión en infraestructura (rampas, botones braille, señalización, etc.) (46,5\%), y una mejor difusión de los principios y alcances de la LIE (27\%).

\section{Discusión de resultados}

Los resultados de la encuesta permiten evidenciar disposiciones diferentes frente a ciertos aspectos de la LIE y el nuevo sistema de admisión (SAE) que operacionaliza sus principios inclusivos. En principio, se constata un alto grado de compromiso con los principios generales que promueve esta nueva regulación. Existe acuerdo respecto de que la ley promueve principios justos, como terminar con la selección de estudiantes y el copago de las familias. No obstante, se aprecian resistencias que, aunque no son mayoritarias, se corresponden con las 
de directoras y directores de escuelas particular subvencionadas de la muestra. Específicamente, existe casi un tercio de directoras/es de esa dependencia que manifiesta una disposición resistente al término del copago de las familias. Es probable que esta se relacione con la percepción de que este desembolso de las familias genera compromisos y responsabilidades que la gratuidad podría poner en tensión, muy en consonancia con las lógicas del mercado educativo que ha regulado el sistema escolar chileno en las últimas décadas.

Adicionalmente, un poco más de la mitad cree que el nuevo sistema de admisión (SAE) afectará el derecho de las familias a elegir escuelas; no obstante, la mayoría absoluta de los encuestados cree que este tipo de sistemas de admisión reduce la discriminación social. En este sentido, las directoras y directores consideran que la nueva regulación se orienta a la justicia social, pues la relacionan con la no discriminación y el aumento de la diversidad del alumnado. A nivel declarativo, se reitera el compromiso con estas medidas, lo que remarca que existe una percepción generalizada de que las discriminaciones sociales a los estudiantes atentan contra la justicia social.

Cabe mencionar que el nuevo sistema de admisión (SAE) no reduce la capacidad de las familias de elegir escuelas; por el contrario, las aumenta, pues al derribar las barreras de selección académica y económica, permite que familias que antaño no podían acceder a una escuela con copago ahora sí puedan hacerlo. Además, el nuevo sistema de admisión prioriza la asignación de escuelas con ciertos criterios. Entre ellos, que cada escuela reciba, al menos, a un 15\% de estudiantes prioritarios o vulnerables (Carrasco, 2018). No obstante esta aclaración, los directivos perciben el nuevo sistema de admisión como un mecanismo que limita la libertad de elegir de las familias. Es probable que en esta opinión adquiera más peso la discusión mediática que ha existido en Chile en torno a la ley, más que una información institucional u oficial.

Respecto de la composición social del alumnado, la mayoría de las entrevistadas y entrevistados considera que la eliminación de barreras selectivas que determina la LIE significará el aumento de estudiantes con necesidades educativas especiales en sus escuelas. Se podría leer este dato como una disposición un tanto crítica o resistente, pues más adelante advierten que no cuentan con las capacidades profesionales para atender a un público más diverso desde esta perspectiva. Otra lectura posible es que la desegregación o la mixtura social no son conceptos visibles para las directoras y directores y que, tal como indican Capper y Young (2014), estas no están familiarizadas o no han incorporado una noción de justicia social que problematice las lógicas de desigualdad escolar.

La asociación de inclusión con niños con necesidades educativas especiales es reduccionista, pero se comprende en el marco de una larga tradición de políticas de inclusión que ha puesto el acento en los déficits de los estudiantes y en entender la diversidad como un proceso de agregación de niños diferentes o que no responden a los patrones convencionales de la escuela, más que en el reconocimiento de las múltiples identidades socioculturales de los sujetos (Rojas, 2018; Rojas y Armijo, 2016).

Respecto de la asociación entre la LIE y los resultados educativos de los estudiantes, existen disposiciones encontradas. Predomina un cierto conformismo, pues la mayoría de los encuestados no vincula la nueva regulación con la calidad de los resultados académicos. Una política de desegregación tendría efectos en la composición del alumnado, pero no necesariamente en las lógicas de aprendizaje. La LIE se fundamenta, en parte, en que la mixtura social del alumnado potencia el efecto par, es decir, las ventajas positivas que se derivan para el aprendizaje de niños y nińas cuando las aulas son más heterogéneas social y académicamente (Bellei, 2013). No obstante, esto no resulta evidente para la mayoría de las directoras y directores que contestaron la encuesta. Hay un grupo menor que cree que sus resultados empeorarán y, probablemente resisten cualquier cambio por los riesgos que ello supone para los resultados en las pruebas nacionales, mientras que $22 \%$ cree que sus resultados mejorarán. Estos últimos tienden a ser directivos de las escuelas de sectores medios-bajos de la muestra, que probablemente confían en que la mixtura social del estudiantado podría llegar a favorecerlos en el tiempo. En ese sentido, podrían manifestar una disposición más comprometida con los alcances de la ley para los aprendizajes. En cambio, entre quienes perciben que sus resultados empeorarán debido a la implementación de la LIE se 
encuentran aquellos directivos que educan a hijos de familias de sectores medios. Es posible que estos prefieran mantener la actual composición social de la escuela, dado el eventual riesgo de la incorporación de estudiantes con un menor capital económico, social y cultural.

Por el contrario, lo que sí es una idea predominante en las directoras y directores es que una política de inclusión es contraria a la lógica de las pruebas estandarizadas con las que se mide la calidad. Aunque no les afecte directamente, lo avizoran como una contradicción. Por una parte, esto puede indicar compromiso con leyes que promuevan a inclusión pero, por otra, resistencia hacia esta nueva regulación si ello no va aparejado con cuestionar la lógica de las pruebas estandarizadas que miden la calidad educativa. El liderazgo directivo orientado a la inclusión y la justicia social se ve condicionado por razones externas e internas a la escuela (Ryan, 2006). Entre las razones externas, destaca la existencia de evaluación estandarizada asociada al financiamiento de las escuelas. Esto se reconoce con fuerza en la encuesta. La presión por los resultados académicos parece incongruente con una demanda por mayor inclusividad. Este es un tema central acerca de la posibilidad de promover políticas inclusivas en un sistema de aseguramiento de la calidad con altas consecuencias como es el chileno. La literatura internacional revisada da cuenta de una tensión profunda entre los empeńos por avanzar en liderazgos que promuevan la justicia social y los sistemas de accountability con altas consecuencias (Anderson, 2009). Ello, porque la presión por los resultados académicos condiciona el profesionalismo docente y resta espacios de autonomía e innovación para planificar la enseñanza teniendo en consideración la diversidad social, étnica, cognitiva, de género y etaria de los estudiantes. Por otra parte, la demanda por una formación ética y moral, que sostiene el enfoque de liderazgo inclusivo y para la justicia social, no pone su centro en los aprendizajes académicos, sino más bien en el proceso de crear un clima de convivencia democrática al interior de la escuela.

Los resultados de la encuesta también ilustran las dificultades y tensiones que observan los directivos. Por una parte, se alude a las capacidades de profesores y profesoras para enfrentar pedagógicamente ambientes de aulas diversas socialmente. Hay acá una disposición crítica acerca de la preparación académica y profesional del profesorado por parte de quienes dirigen las escuelas. Esto muestra que existe poca confianza en los docentes de parte de sus propios directivos, y, además, que podría ser muy complejo liderar procesos de desegregación e inclusión social con un cuerpo profesional que se evalúa con debilidades en el tema. De hecho, los directivos apuestan por aumentar el apoyo de profesionales del área de la psicología y la psicopedagogía como recurso más especializado en temas de diversidad social. En este punto, la literatura refiere que la inclusión social es un objetivo de toda la comunidad escolar (directivos, profesores, padres, estudiantes, etc.), no solo de un tipo de profesional especializado (Blackmore, 2009; Shields, 2004).

\section{Conclusiones}

La Ley de Inclusión es el ejemplo de una política de desegregación que interviene mecanismos de mercado que están a la base de los procesos de segmentación social del sistema escolar chileno, como la selección escolar y el copago (Bellei, 2013). Su acción opera fundamentalmente contra las barreras de acceso a las escuelas, estipulando simultáneamente que niños y niñas no pueden ser expuestos a discriminaciones arbitrarias. Por lo mismo, la ley establece límites para la suspensión y la expulsión de estudiantes. Estas discriminaciones arbitrarias poseen una definición amplia, que involucra temas como la discriminación derivada del racismo, el sexismo, el clasismo, las diferencias etarias, los prejuicios hacia las personas con capacidades diferentes, las creencias religiosas, entre otras. La concepción amplia de esta definición subraya que la inclusión alude a dimensiones múltiples y de ninguna manera se reduce a una sola condición de los sujetos. Fomentar la diversidad y mixtura del alumnado es un referente de justicia en la escuela, pues avanza en la antigua discusión sobre la distribución igualitaria de oportunidades y condiciones para educar a todos los niños y niñas sin que sus orígenes sociales incidan en la educación que les ofrece el Estado y la sociedad (Ayed, 2015). 
Estos principios son aceptados por las directoras y directores de escuelas que han comenzado a vivir la implementación reciente de la Ley de Inclusión en Chile. Predomina una disposición de compromiso con estos postulados generales que promueven el fin de toda discriminación. No obstante, la investigación permite evidenciar zonas de conformismo y resistencia de parte de los directivos por la falta de condiciones para la implementación de una regulación de esta naturaleza, destacando entre ellas la fragilidad de los recursos pedagógicos y profesionales, y la mantención de una lógica evaluativa de la calidad que apunta a la estandarización de los resultados académicos, principio contrario a la diversidad y la inclusión.

En este escenario, parece necesario que quienes lideran las escuelas cuenten con espacios formativos para reflexionar sobre los alcances de una política de desegregación y, fundamentalmente, entender la relación que esta tiene con la mixtura social, la diversidad y la justicia. Para movilizar estos conceptos, los actores escolares requieren condiciones para incorporarlos más complejamente, reflexionar sobre ellos y trabajarlos con sus comunidades.

Una política de esta envergadura requiere de un sistema de comunicación y difusión de parte de los gobiernos que sea intensivo, efectivo y explicite los fines que pretende desencadenar. De lo contrario, se corre el riesgo de que esta ley sea incorporada como una regulación más de las múltiples políticas públicas con la que los directivos deben lidiar cotidianamente. Es más, este estudio propone que un porcentaje importante de los directivos comprende esta ley como una prolongación de las políticas que regulan la integración de nińos con necesidades educativas especiales a las escuelas, asociando con ello la desegregación escolar con el aumento de alumnos y alumnas que requieren atención psicopedagógica especializada. Esta forma de comprensión es coherente con las racionalidades que han imperado en las políticas educativas de estos últimos 30 años en Chile. La política es apreciada como dispositivo de reparación de los sujetos individuales y de las debilidades que portan, y no como un dispositivo que repara desigualdades sociales, que no son responsabilidades de los sujetos, sino de inequidades estructurales que se expresan en los desempeños de niños y niñas. Tal como afirman Oplatka \& Arar (2015), directoras y directores se percibirán como protagonistas de la implementación de la Ley de Inclusión en la medida en que posean las condiciones para construir una conciencia crítica respecto de las desigualdades sociales del sistema escolar.

El artículo original fue recibido el 17 de diciembre de 2019

El artículo revisado fue recibido el 30 de diciembre de 2019

El artículo fue aceptado el 9 de marzo de 2020

\section{Referencias}

Anderson, G. (2009). Advocacy Leadership Toward a Post-Reform Agenda in Education. Nueva York, NY: Routledge.

Archambault, J. \& Richer, C. (2014). Leadership for Social Justice Throughout Fifteen Years of Intervention in a Disadvantaged and Multicultural Canadian Urban Area: The Supporting Montréal Schools Program. En I. Bogotch \& C. Shields (Eds.), International Handbook of Educational Leadership and Social (In)Justice (pp. 1023-1045). Nueva York, NY: Springer.

Arcidiácono, M., Cruces, G., Gasparini, L., Jaume, D., Serio, M., y Vázquez, E. (2014). La segregación escolar públicoprivada en América Latina. Santiago, Chile: Naciones Unidas-CEPAL.

Ayed, C. B. (2015). La mixité sociale á l'école. Tensions, enjeux, perspectives. París, Francia: Armand Colin.

Ball, S., Maguire, M., \& Braun, A. (2012). How Schools Do Policy: Policy Enactments in Secondary Schools. Nueva York, NY: Routledge

Blackmore, J. (2009). Leadership for Social Justice: A Transnational Dialogue. Journal Of Research On Leadership Education, 4(1), 1-10. https://doi.org/10.1177/194277510900400105 
Bellei, C. (2013). El estudio de la segregación socioeconómica y académica de la educación chilena. Estudios Pedagógicos, 39(1), 325-345. https://doi.org/10.4067/S0718-07052013000100019

Biblioteca del Congreso Nacional (BCN). (2014). Historia de la Ley $N^{\circ} 20.845$. De inclusión escolar que regula la admisión de los y las estudiantes, elimina el financiamiento compartido y prohibe el lucro en establecimientos educacionales que reciben aportes del estado. Santiago, Chile: Biblioteca del Congreso Nacional.

Bogotch, I. \& Reyes-Guerra, D. (2014). Leadership for Social Justice: Social Justice Pedagogies. Revista Internacional De Educación Para La Justicia Social (RIEJS), 3(2), 33-58. Recuperado de http://hdl.handle.net/10486/666735

Capper, C. \& Young, M. (2014). Ironies and Limitations of Educational Leadership for Social Justice: A Call to Social Justice Educators. Theory Into Practice, 53(2), 158-164. https://doi.org/10.1080/00405841.2014.885814

Carrasco, A. (2018). Nuevo sistema de admisión escolar y elección de escuela: ‘aseguran calidad? En I. Sánchez (Ed.), Ideas de Educación II. Definiciones en tiempos de cambio (pp. 167-197). Santiago, Chile: Ediciones UC.

Castillo, J. (2017). El proceso de politización como contexto de la ley de inclusión escolar. En Ministerio de Educación, El primer gran debate de la reforma educacional: Ley de Inclusión Escolar (pp. 26-47). Santiago, Chile: Ministerio de Educación. Recuperado de https://hdl.handle.net/20.500.12365/441

Draelants, H. (2018). Comment l'école reste inégalitaire. Comprendre pour mieux réformer. Lovaina, Bélgica: Presses Universitaires de Louvain.

Elacqua, G. (2012). The impact of school choice and public policy on segregation: Evidence from Chile. International Journal Of Educational Development, 32(3), 444-453. https://doi.org/10.1016/j.ijedudev.2011.08.003

Ministerio de Educación (MINEDUC). (2012). Serie Evidencias: Medidas de segregación escolar: discusión para el caso chileno. Santiago, Chile: Centro de Estudios - MINEDUC.

Ministerio de Educación (MINEDUC). (2017). Resumen Estadístico de la Educación 2017. Santiago, Chile: Centro de Estudios - MINEDUC.

Molla, T. \& Gale, T. (2018). Positional matters: school leaders engaging with national equity agendas. Journal of Educational Policy, 34(6), 858-876. https://doi.org/10.1080/02680939.2018.1556811

Murillo, F. y Martínez-Garrido, C. (2017). Estimación de la magnitud de la segregación escolar en América Latina. Magis. Revista Internacional de Investigación en Educación, 9(19), 11-30. https://doi.org/10.11144/javeriana.m9-19.emse

Lipsky, M. (2010). Street-Level Bureaucracy: Dilemmas of the Individual in Public Services (30th Anniversary Expanded Edition). Nueva York, NY: Russell Sage Foundation.

OECD. (2012). Equity and Quality in Education. Supporting Disadvantaged Students and Schools. París, Francia: OECD Publishing.

OECD. (2018). Education in Chile, Reviews of National Policies for Education. París, Francia: OECD Publishing.

Oplatka, I. \& Arar, K. (2015). Leadership for social justice and the characteristics of traditional societies: ponderings on the application of western-grounded models. International Journal Of Leadership In Education, 19(3), 352-369. https://doi.org/10.1080/13603124.2015.1028464

Rojas, M. T. (2018). Inclusión social: miradas de los docentes y apoderados frente a la mixtura social en sus escuelas. Estudios Pedagógicos, 44(3), 217-234. https://doi.org/10.4067/S0718-07052018000300217

Rojas, M. T. y Armijo, M. (2016). Qué es la inclusión escolar: distintas perspectivas en debate. Cuadernos De Educación, (75), 1-11.

Ryan, J. (2006). Inclusive Leadership and Social Justice for Schools. Leadership And Policy In Schools, 5(1), 3-17. https://doi.org/10.1080/15700760500483995

Ryan, J. (2010). Promoting social justice in schools: principals' political strategies. International Journal Of Leadership In Education, 13(4), 357-376. https://doi.org/10.1080/13603124.2010.503281

Santos, H. \& Elacqua, G. (2017). Socioeconomic school segregation in Chile: Parental choice and a theoretical counterfactual analysis. CEPAL Review, 2016(119), 123-137. https://doi.org/10.18356/938a3490-en

Shields, C. (2004). Dialogic Leadership for Social Justice: Overcoming Pathologies of Silence. Educational Administration Quarterly, 40(1), 109-132. doi: 10.1177/0013161x03258963

Theoharis, G. (2010). Disrupting injustice: Principals narrate the strategies they use to improve their schools and advance social justice. The Teachers College Record, 112(1), 331-373. Recuperado de https://www.tcrecord.org/Content.asp?ContentId=15842 